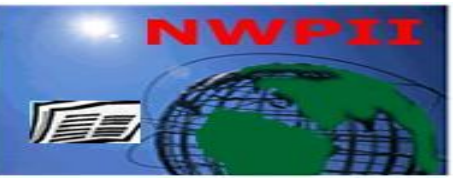

American Journal of Biomedical Sciences

ISSN: 1937-9080

nwpii.com/ajbms

\title{
Radiofrequency Dosimetry in Subjects Implanted with Metallic Structures Undergoing MRI: a Numerical Study
}

\author{
E Mattei ${ }^{1}$, M Triventi ${ }^{1}$, G Calcagnini ${ }^{1}$, F Censi $^{1}$, and P Bartolini ${ }^{1}$ \\ ${ }^{1}$ Department of Technologies and Health, Italian National Institute of Health, Roma, Italy \\ *Corresponding Author: \\ Eugenio Mattei \\ Italian National Institute of Health \\ Viale Regina Elena 299 \\ Rome, Italy \\ Email: eugenio.mattei@iss.it
}

Received: March 27, 2009; | Revised: June 21, 2009; | Accepted: July 14, 2009

\begin{abstract}
A numerical study to investigate the effects of the exposure to radiofrequency (RF) field on biological tissues implanted with thin metallic structures has been carried out, using the finite difference time domain (FDTD) solution technique. The particular case of the RF used in the clinical $1.5 \mathrm{~T}$ magnetic resonance imaging (MRI) scanners has been studied. The results of the model show that the presence of a metallic wire yields to a significant increase in the local specific absorption rate (SAR) and in temperature around the implant. Present standards or guidelines do not specifically address this problem and use threshold levels and methods to define safe exposure conditions that cannot apply to reveal high SAR gradients, such as the ones generated by thin metallic implanted objects. In particular, an averaging mass of $10 \mathrm{~g}$, typically used for SAR calculation to evaluate the effect of RF exposure of biological tissues, is too big if compared to the SAR gradient generated by thin metallic structures. The SAR estimation according to this method cannot be considered a reliable indicator to evaluate the potential damage due to the RF induced heating at the interface between the implant and biological tissues. Five wires of different lengths and thicknesses were simulated and the SAR at the wire tip was evaluated over $10 \mathrm{~g}, 1 \mathrm{~g}, 0.1 \mathrm{~g}$ averaging mass: the SAR underestimation related to the $10 \mathrm{~g}$ and $1 \mathrm{~g}$ masses, compared to $0.1 \mathrm{~g}$ mass, was greater than $90 \%$ and $60 \%$, respectively. In conclusion, present standards cannot be applied to thin metallic implants. The amount of averaging mass should be chosen as a function of the dimension of the implanted object and of the SAR gradient that is generated around it. At the same time, the averaging volume has to be not too small to be just a single "hot spot", not relevant for the evaluation of potential tissue damage due to RF exposure.
\end{abstract}

Keywords: MRI; RF field; RF heating; SAR; implantable devices 


\section{Introduction}

Radiofrequency (RF) induced heating of biological tissue has long been a concern for patients undergoing magnetic resonance imaging (MRI), leading to national and international standards intended to regulate patient exposure and minimize the risk [1,2]. The specific absorption rate (SAR, defined as the amount of RF power absorbed per unit of mass of an object $\left.\left(\mathrm{Wkg}^{-1}\right)\right)$ is the current standard for characterizing the thermogenic aspects of this electromagnetic field. As originally applied, however, such standards were designed for heating in biological tissue devoid of metallic or conductive implants. According to the International Commission on Non-Ionizing Radiation Protection (ICNIRP), in the frequency range from $100 \mathrm{kHz}$ to $10 \mathrm{GHz}$, the limit for head and trunk is $10 \mathrm{Wkg}^{-1}$ averaged over any $10 \mathrm{~g}$ mass of tissue for occupational situations; for the general public, this value goes down to $2 \mathrm{Wkg}^{-1}$, always averaged over any $10 \mathrm{~g}$ mass of tissue. For patients undergoing an MRI examination, ICNIRP guidelines provide specific SAR exposure limits, as a function of the duration of the scan and of the particular body district where the RF power is deposited. In general a whole-body SAR of $2 \mathrm{Wkg}^{-1}$ is the standard level that is not exceed during typical MRI procedures. The case of MRI investigations is addressed in a particular ICNIRP document [3] where SAR threshold levels are increased up to $4-12 \mathrm{Wkg}^{-1}$, as a function of the body district of interest. However, the case of implanted patient is not covered. Therefore, such limits cannot be immediately extended also to patients implanted with metallic wires and/or active medical devices. Since, to date, no other standard specifically addresses this issue, further studies and several considerations are needed.

When a patient implanted with conductive structures is exposed to electromagnetic fields (EMF), the metallic object may operate as an antenna which causes an increase in power deposition around the wire or catheter. With regards to the RF field generated by an MRI scanner, several studies have investigated, both in vivo and in vitro, the potential adverse effects on pacemakers (PM), implantable cardioverters
(ICD), brain stimulator (BS), cochlear implants (CI), stent [4-6] and other similar implantable devices. This increase in the local SAR is generally limited to a very small region (comparable to the dimensions of the metallic wire), so that the induced temperature increase is unlikely to produce dangerous systemic effect for the patient. However, harmful effects in the region the surrounds the metallic implant might still occur and modify the interface between the implant and biological tissues. As a consequence, if the implanted device implies energy transfer towards biological tissues (as for the case of leads used in PM, ICD, CI, BS, etc.) the correct behaviour of the device could not be guaranteed anymore.

In this study we numerically reproduce an EMF exposure at $64 \mathrm{MHz}$ (RF field used by $1.5 \mathrm{~T}$ MRI clinical scanners) that results in maximum SAR values below the safety thresholds of the present international standards and guidelines, on a rectangular phantom which simulates biological tissues, implanted with thin metallic wires.

Aims of the paper are:

1. to show the effect of thin metallic structures on the local distribution of the electric field and the associated SAR;

2. to determine to what extent the present standard methods for SAR evaluation are appropriate in the presence of thin metallic structures;

3. to propose a refinement of the actual standard to meet the constraint of this particular application.

\section{Methods and materials}

We developed a simple numerical model in order to calculate the SAR distribution inside a homogeneous domain, with electromagnetic properties similar to those of biological tissues, implanted with metallic wires (Figure 1). The model was exposed to a combination of plane waves, able to generate a pure circular polarized magnetic field [7], that is the typical exposure used during MRI investigations. We used commercial FDTD (Finite Difference Time Domain) software (SEMCAD X, version 11.0, SPEAG, Zurich) that incorporated native drawing. The software had a variable grid (graded-mesh) generator and was used on a personal computer (C) 2009 by NWPII. All rights reserved. 


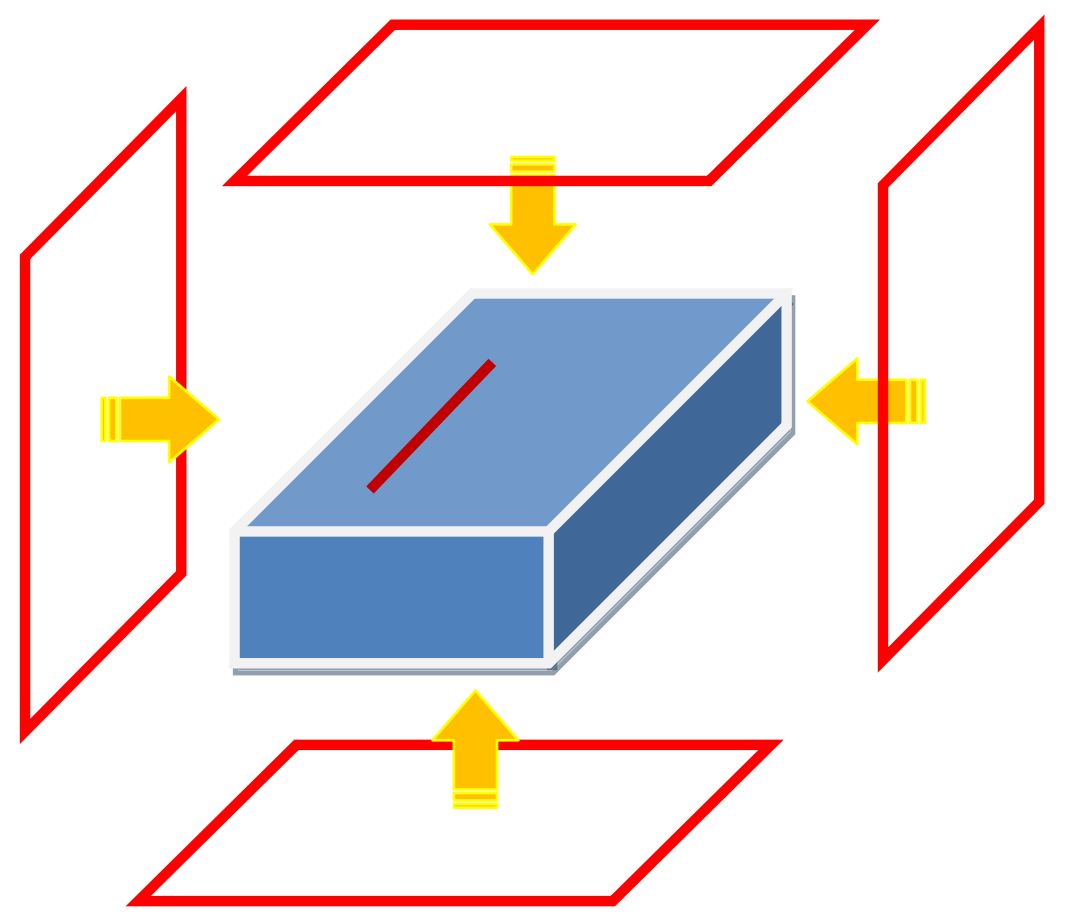

Figure 1. Schematic representation of the numerical model. The exposure conditions are obtained using two couples of plane waves; in each couple, the plane waves propagate on parallel but opposite directions, with concordant B vector component and opposite E vector component. Between the two couples, a $90^{\circ}$ shift both in space and in time was applied. A circular-polarized magnetic field is thus reproduced.

Table I. Physical properties of the dielectric material used in the numerical model to simulate biological tissues.

Properties Value@64 MHz Unit

\begin{tabular}{ccc}
\hline \hline Rel. Permittivity & 79 & - \\
Rel. Permeability & 1 & - \\
Electrical Conductivity & 0.6 & $\mathrm{~S} \mathrm{~m}^{-1}$ \\
Magnetic Conductivity & 0 & $\mathrm{Ohm} \mathrm{m}^{-1}$ \\
Density & 1007.5 & $\mathrm{Kg} \mathrm{m}^{-3}$ \\
Specific Heat Capacity & 4178.6 & $\mathrm{~J} \mathrm{~kg} \mathrm{~K}^{-1}$ \\
Thermal Conductivity & 0.2 & $\mathrm{~W} \mathrm{~m}^{-1} \mathrm{~K}^{-1}$ \\
\hline \hline
\end{tabular}

The frequency we tested is $64 \mathrm{MHz}$, corresponding to the RF used by the MRI scanners today most used in the clinical practice, which work with a static magnetic field of $1.5 \mathrm{~T}$. The plane waves propagate inside a $610 \mathrm{~mm} \times 350 \mathrm{mmx} 115 \mathrm{~mm}$ box, drawn by using the native 3-D modelling section of the software and simulating a homogeneous portion of biological 
tissues. The physical properties assigned to this domain are reported in Table I. A rectangular plastic box filled with saline solution has been widely used in both numerical and in-vitro studies on electromagnetic interference to simulate the human trunk [8-10]. Inside the box we placed metallic wires of different thickness and length:

1) wire 1: diameter $=1 \mathrm{~mm}$; length $=25 \mathrm{~cm}$;

2) wire 2: diameter $=2 \mathrm{~mm}$; length $=25 \mathrm{~cm}$;

3) wire 3: diameter $=3 \mathrm{~mm}$; length $=25 \mathrm{~cm}$;

4) wire 4: diameter $=2 \mathrm{~mm}$; length $=15 \mathrm{~cm}$;

5) wire 5: diameter $=2 \mathrm{~mm}$; length $=40 \mathrm{~cm}$;

The thickness of the wires was chosen according to what are the typical dimensions of a metallic implant, such as a PM, ICD or brain stimulator lead. A $25 \mathrm{~cm}$-long wire is close to the theoretical resonance length at $64 \mathrm{MHz}$ inside the dielectric domain we modelled; $15 \mathrm{~cm}$ and 40 represent a condition of an implant far from its resonance length, too short or too long, respectively. All the wires were positioned $2 \mathrm{~cm}$ far from the side surfaces of the box.

The graded mesh software allowed us to generate a non-uniform grid for the FDTD solution: at the lead tip, the mesh was generated with a fixed step of $0.25 \mathrm{~mm}$. Over the area covered by the implant a minimum step of 0.5 $\mathrm{mm}$, a maximum step of $5 \mathrm{~mm}$ and a growth factor of 1.3 were used. In the other parts of the model the grid parameters are: $1 \mathrm{~mm}$ minimum step; 50 $\mathrm{mm}$ maximum step; 1.3 growing factor. The duration of the RF exposure was 10 periods, with an initial ramped interval of 2 periods. SEMCAD implements a particular algorithm for the SAR extraction according to the IEEE P1529 standard (Recommended Practice for determining the spatial-peak SAR associated with the use of wireless handsets - computational techniques) [11]. This algorithm allows computing the spatialpeak SAR over any required mass. All different tissue types, organs or parts of the model can be selected for the averaging process.

We performed preliminary simulations without the wire, in order to set the amplitude of the plane waves to produce inside the dielectric domain a whole-body mean SAR of $2 \mathrm{~W} \mathrm{~kg}^{-1}$, (maximum value accepted in standard MRI clinical investigations). The FDTD grid adopted in these simulations was the same used in the following numerical studies, where a metallic implants was placed inside the dielectric material: a much finer grid was thus produced in the area where the metallic wires will be located, so to ensure a correct comparability for the results computed.

Keeping the amplitude of the plane waves constant, we repeated the simulations with the wires placed inside the dielectric domain. We calculated the values of SAR averaged over $1 \mathrm{~g}$ $\left(\mathrm{SAR}_{1 \mathrm{~g}}\right)$ and $10 \mathrm{~g}\left(\mathrm{SAR} 1_{0 \mathrm{~g}}\right)$, using the IEEE P1529 algorithm. In order to evaluate the SAR field distribution in close proximity to the wire tip surface, we averaged the local SAR over $0.1 \mathrm{~g}$ mass $\left(\mathrm{SAR}_{0.1 \mathrm{~g}}\right)$ of the dielectric material. Given the physical properties of the domain used to simulate biological tissues, a $10 \mathrm{~g}$ mass corresponds to a cubic region of $22 \times 22 \times 22 \mathrm{~mm}^{3}, 1$ $\mathrm{g}$ mass to a $10 \times 10 \times 10 \mathrm{~mm}^{3}$ region, $0.1 \mathrm{~g}$ mass to a $5 \times 5 \times 5 \mathrm{~mm}^{3}$.

Additional simulations were performed increasing the number of voxel in the model, in order to be sure that a finer mesh does not affect any of the parameters we used in our analysis.

For the five wires, the SAR gradient has been computed and compared: as outcome of the study, we want to identify a possible relation between the implant dimensions and the SAR gradient, so to find the optimal averaging mass as a trade-off between a volume small enough to cover significantly high SAR value and, at the same time, big enough to avoid the case of a local "hot spot", not relevant for the tissue damage.

Always using the same grid, we then coupled the electromagnetic solution with a thermal analysis inside the dielectric domain. As done for the SAR calculation, we first computed the temperature increase without the wire; then we investigated how the presence of the metallic implant might change the temperature distribution. With regard to the thermal properties assigned to the dielectric domain, we reproduced a worse-case condition, since we assumed the conduction as the only contribute to the heat transfer (no convection).

The thermal simulation was performed for a total time of $2000 \mathrm{~s}$, with the RF source active after $200 \mathrm{~s}$ to the end. 


\section{Results}

The plane waves at $64 \mathrm{MHz}$ used as RF source produced a maximum $\mathrm{SAR}_{10 \mathrm{~g}}$ of $7.6 \mathrm{Wkg}^{-1}$ inside the dielectric material, without the metallic wire. If averaged over $1 \mathrm{~g}$ or $0.1 \mathrm{~g}$, no significant changes were observed in the maximum SAR value computed $\left(7.1 \mathrm{Wkg}^{-1}\right.$ and $7.8 \mathrm{Wkg}^{-1}$, respectively).

The electromagnetic pattern deeply changes when a metallic implant is placed inside the box. In Figure 2 we reported the E field distribution inside the dielectric domain, with and without the metallic implants, for the five wires we studied.
Figure 3 shows the SAR distribution resulting from the numerical model of the dielectric material implanted with the $2 \mathrm{~mm}$-thick wire. The increase in the local SAR is detectable either if using the $10 \mathrm{~g}, 1 \mathrm{~g}$ and $0.1 \mathrm{~g}$ averaging mass, even if the estimated maximum deposited power at the lead tip markedly differs $\left(\mathrm{SAR}_{10 \mathrm{~g}}=44.0 \mathrm{Wkg}^{-1}\right.$, $\left.\mathrm{SAR}_{1 \mathrm{~g}}=197.0 \mathrm{Wkg}^{-1}, \mathrm{SAR}_{0.1 \mathrm{~g}}=737.1 \mathrm{Wkg}^{-1}\right)$. In order to compare the averaging volumes with the dimensions of the SAR gradient, we plotted over the power distribution resulting around the lead tip, the cubic regions corresponding to $10 \mathrm{~g}, 1 \mathrm{~g}$ and $0.1 \mathrm{~g}$ mass. Figure 4 refers to the $2 \mathrm{~mm}$-thick, $25 \mathrm{~cm}$-long wire.
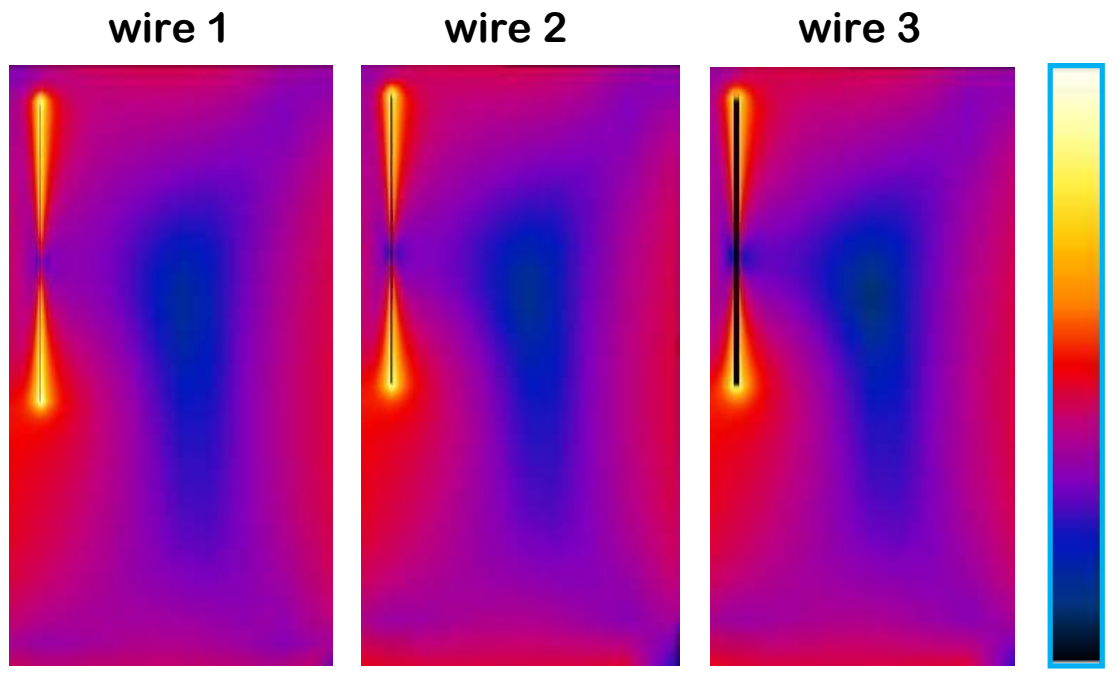

0 dB normalized $-5$ to: $2 \mathrm{e}^{3} \mathrm{~V} \mathrm{~m}^{-1}$

wire 4

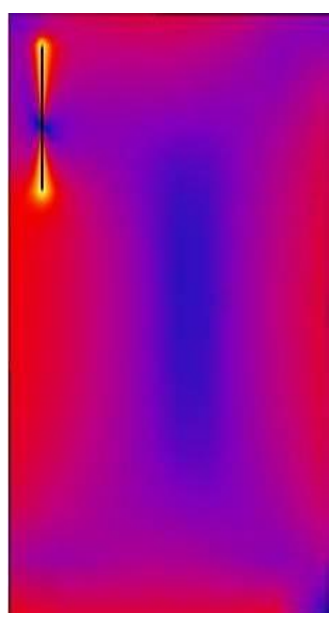

wire 5
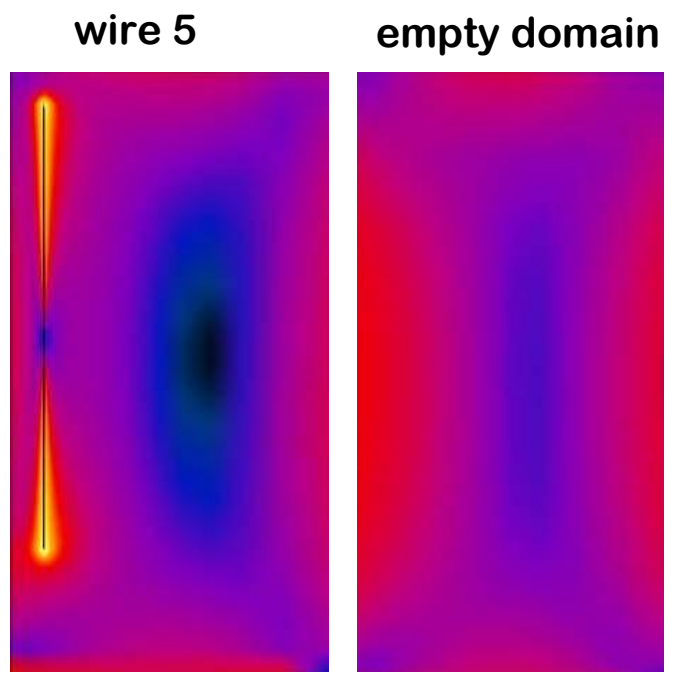
Figure 2. E field pattern inside the dielectric domain. Wire 1: diameter $=1 \mathrm{~mm}$; length $=25 \mathrm{~cm}$; wire 2 : diameter $=2$ $\mathrm{mm}$; length $=25 \mathrm{~cm}$; wire 3 : diameter $=3 \mathrm{~mm}$; length $=25 \mathrm{~cm}$; wire 4 : diameter $=2 \mathrm{~mm}$; length $=15 \mathrm{~cm}$; wire 5 : diameter $=2 \mathrm{~mm}$; length $=40 \mathrm{~cm}$.

In figure 5, a, the $\mathrm{SAR}_{10 \mathrm{~g}}, \mathrm{SAR}_{1 \mathrm{~g}}$ and $\mathrm{SAR}_{0.1 \mathrm{~g}}$ are compared for the five wires we modelled. Keeping the SAR value averaged over $0.1 \mathrm{~g}$ as reference, we calculated the underestimation related to bigger averaging masses $(10 \mathrm{~g}$ and $1 \mathrm{~g})$, for the three thicknesses and the three lengths we simulated : the results are presented as percentage underestimation in figure $5, \mathrm{~b}$. a) SAR $_{10 \mathrm{~g}}$

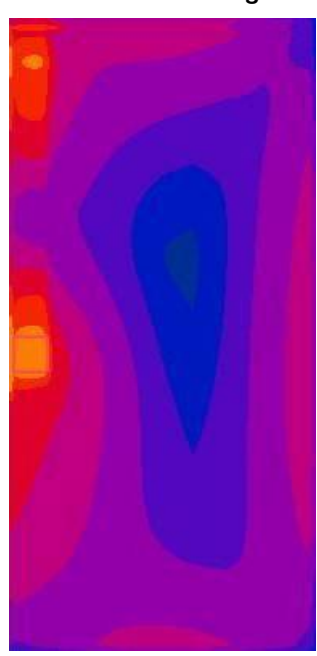

b) $S_{19}$

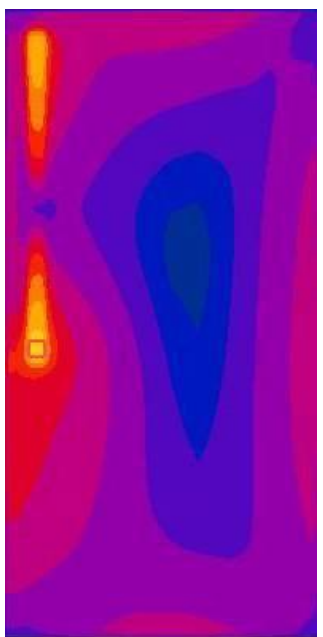

We also compared the dimensions of the SAR gradients: the graphs reported in figure 6, a, show the SAR exponential decrease along a line parallel to the wire, starting from the tip towards the dielectric domain. For the $1 \mathrm{~mm}$-thick wire, SAR goes down to the $20 \%$ of the maximum value at about $2.5 \mathrm{~mm}$ from the metallic surface.
Figure 3. SAR distribution resulting from the numerical model of a dielectric box implanted with the 2 mm-thick, 25 $\mathrm{cm}$-long wire: transversal section through a plane containing the implant. a) SAR averaged over $10 \mathrm{~g}$ mass; b) SAR averaged over $1 \mathrm{~g}$ mass; c) SAR averaged over $0.1 \mathrm{~g}$ mass. a) $10 \mathrm{~g}$ mass

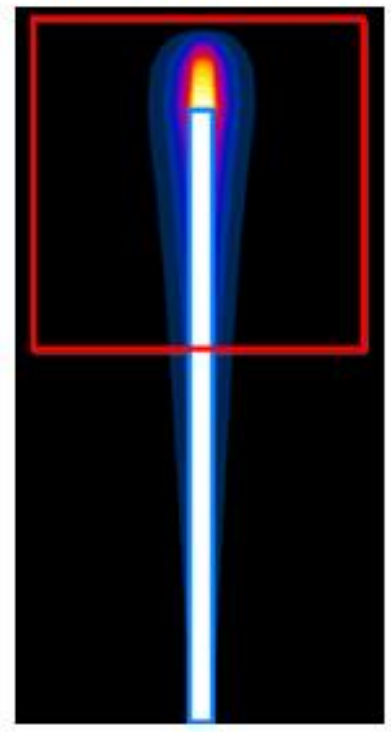

b) $1 \mathrm{~g}$ mass

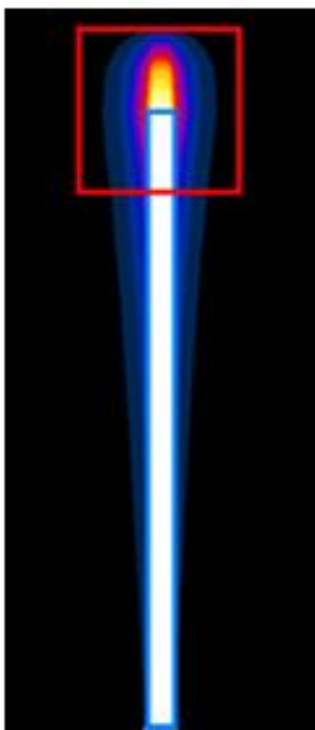

c) $0.1 \mathrm{~g}$ mass

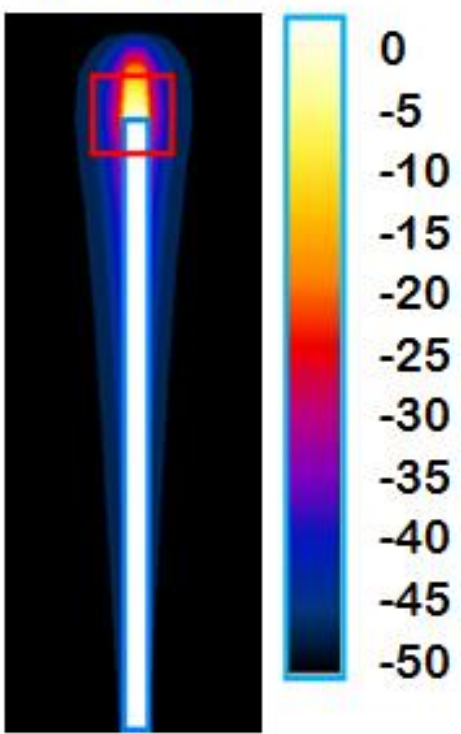

C 2009 by NWPII. All rights reserved.
dB normalized to: $2 \mathrm{e}^{3} \mathrm{~W} \mathrm{~kg}^{-1}$ 
Figure 4. Comparison between the RF induced SAR gradient around the lead tip and the averaging mass use to evaluate the local SAR: a) $10 \mathrm{~g}$ mass; b) $1 \mathrm{~g}$ mass; c) $0.1 \mathrm{~g}$ mass.

As the dimensions of the tip increase, the extent of the SAR peak becomes larger: the $20 \%$ of the maximum value is reached approximately at $3 \mathrm{~mm}$ away for the $2 \mathrm{~mm}$ lead and at $4 \mathrm{~mm}$ away for the $4 \mathrm{~mm}$ lead.

On the other hand, the SAR gradient computed for the wires of different length is much more similar, in spite of quite different maximum SAR value reached at the wire tip (Figure 6, b).
The thermal analysis performed assuming no convection inside the dielectric domain, for a heating period of $1800 \mathrm{~s}$, reveals that the induced current flowing from the metallic lead inside the lossy material generates high temperature increase all around the tip. The heating induced by the RF source in the empty phantom is, on the other hand, rather low $\left(\approx 2^{\circ} \mathrm{C}\right)$.

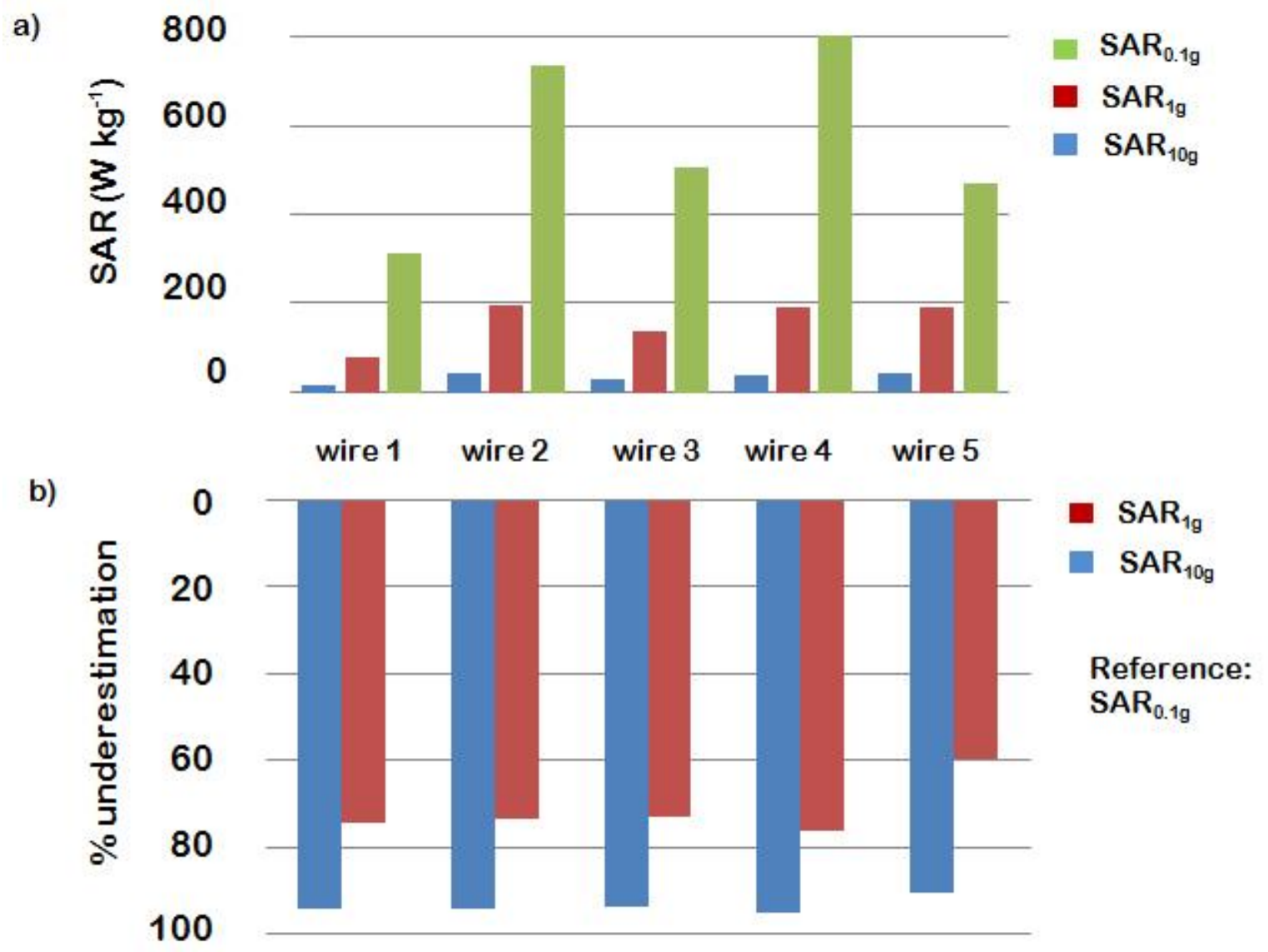

Figure 5. a) Comparison between the maximum SAR computed over different averaging masses. b) Percentage underestimation related to the SAR calculation made on an average volume of $10 \mathrm{~g}$ and $1 \mathrm{~g}$, having as reference the SAR value computed over $0.1 \mathrm{~g}$. Wire 1 : diameter $=1 \mathrm{~mm}$; length $=25 \mathrm{~cm}$; wire 2 : diameter $=2 \mathrm{~mm}$; length $=25 \mathrm{~cm}$; wire 3: diameter $=3 \mathrm{~mm}$; length $=25 \mathrm{~cm}$; wire 4 : diameter $=2 \mathrm{~mm}$; length $=15 \mathrm{~cm}$; wire 5 : diameter $=2 \mathrm{~mm}$; length $=40 \mathrm{~cm}$.

In figure 7 we reported the temperature rises at different distances from the lead tip, for the $1 \mathrm{~mm}, 2$ $\mathrm{mm}$ and $4 \mathrm{~mm}$-thick wires. The highest temperature is obtained for the thinnest wire (temperature increase $\Delta \mathrm{T}=30.8^{\circ} \mathrm{C}$ ), whereas for the $2 \mathrm{~mm}$ and for the $4 \mathrm{~mm}$ lead the temperature increase is as low as $\Delta \mathrm{T}=15.9^{\circ} \mathrm{C}$ and $\Delta \mathrm{T}=9.1^{\circ} \mathrm{C}$, respectively. The tip dimensions Am. J. Biomed. Sci. 2009, 1(4), 373-384; doi: 10.5099/aj090400373 influence also the temperature gradient that rises around the implant: the thicker is the wire, the less is the difference between the temperature profiles sampled at $0 \mathrm{~mm}, 1 \mathrm{~mm}, 2 \mathrm{~mm}$ and $3 \mathrm{~mm}$ away from the tip. On the other hand, when comparing the induced heating for the wires of different length (figure 8), the temperature gradients seem quite similar, in (c) 2009 by NWPII. All rights reserved. 

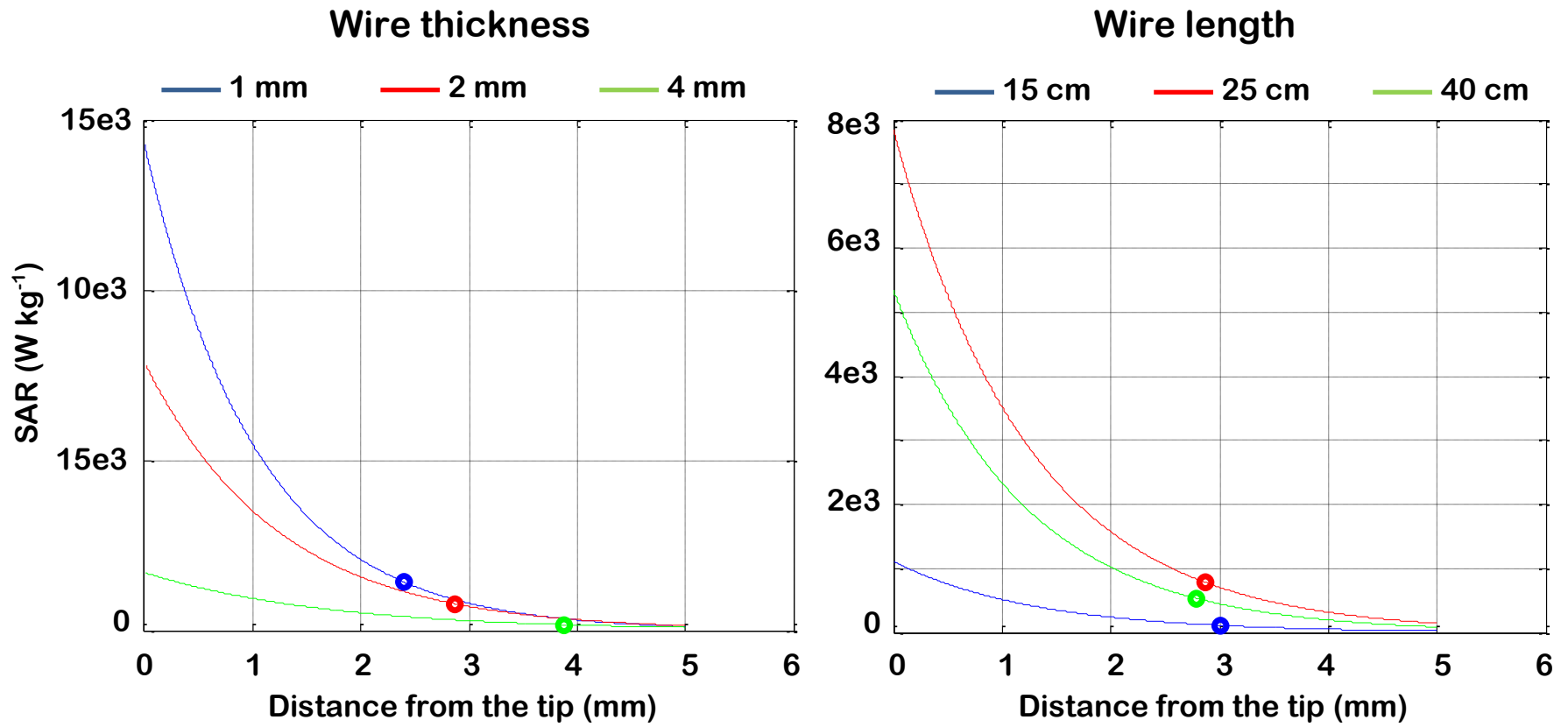

Figure 6. SAR exponential decrease along a line parallel to the wire, starting from the tip towards the dielectric domain. a) $1 \mathrm{~mm}, 2 \mathrm{~mm}$, and $4 \mathrm{~mm}$-thick wires. b) $15 \mathrm{~cm}, 25 \mathrm{~cm}$, and $40 \mathrm{~cm}$-long wires. Circles indicate the distance at which the $20 \%$ of the maximum SAR is reached.

\section{Discussion}

In the $100 \mathrm{kHz}$ to $10 \mathrm{GHz}$ frequency range, a limit of $2 \mathrm{Wkg}^{-1}$ represents the highest threshold below which no significant harmful effects occur to any kind of biological tissues, according to the present international standards. For the exposure of biological tissues to the RF field generated by an MRI scanner, ICNIRP guidelines extend towards higher levels than the general safety values. In the frequency range from $100 \mathrm{kHz}$ to $10 \mathrm{GHz}$ the maximum localized SAR in the head and trunk is limited to $10 \mathrm{~W} \mathrm{~kg}^{-1}$ averaged over any $10 \mathrm{~g}$ mass of tissue, in case of occupational situations; for the general public, this value goes down to $2 \mathrm{Wkg}^{-1}$, always averaged over any $10 \mathrm{~g}$ mass of tissue.

However, such values come out from several studies [12-15] involving general public and cannot be immediately extended also to patients implanted with metallic devices, such as PMs, ICDs, BSs or CIs. In particular, the methods suggested in present standards for the local SAR evaluation do not take into account the presence of thin metallic implants, which might cause very high SAR gradient, and thus could result in misleading evaluations of the deposited power.

In this paper we developed a numerical model to show the effect of thin metallic structures on the local distribution of the electric field and the associated SAR, and to determine to what extent the currently accepted methods for SAR evaluation are appropriate in the presence of thin metallic structures; In addition we propose a criteria to chose an appropriate averaging mass to be used to evaluate the power deposited by thin metallic structures in biological tissues.

The presence of a metallic object may cause an increase in the power deposition which might lead to adverse health consequences. Calculations of RF induced temperature increase for a realistic numerical model of human tissues, in absence of metallic structures, developed both with the finite element or the FDTD technique [16,17], show a maximum rise of about $1{ }^{\circ} \mathrm{C}$ from the absorption of $10 \mathrm{Wkg}^{-1}$. ICNIRP localised SAR limits have been chosen not to exceed such a value, and it is recommended that an averaging mass of $10 \mathrm{~g}$ is used to calculate the induced SAR. Such a value is (C) 2009 by NWPII. All rights reserved. 
chosen to take into account the inhomogeneous spatial distribution of energy absorbed, which may result in significant heating of particular anatomic districts.

When a RF field interacts with tissues implanted with thin metallic structures, it generates very high SAR gradients, which make the spatial distribution of energy absorbed much more inhomogeneous. Inside the metallic object, considered as a perfect conductor, the $\mathrm{E}$ field disappears, whereas outside the field lines bend perpendicular to the surface of the implant. If the surface area is small, a dense electromagnetic flux may arise near the wire. In lossy tissues, this leads to higher power absorption near the implant, compared to the same tissue with no implant. Thus, in case of thin metallic structures, an averaging mass of $10 \mathrm{~g}$ might be too big to reveal

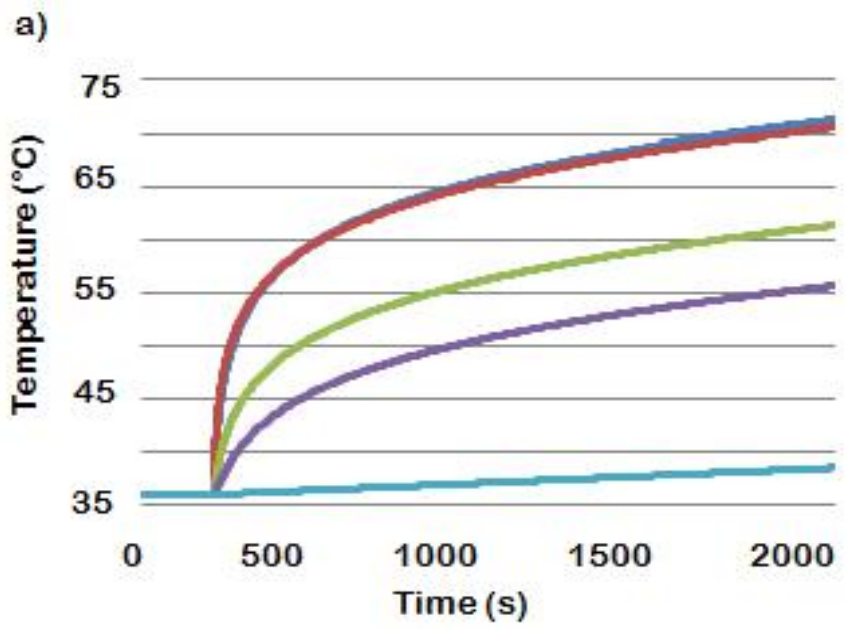

c)

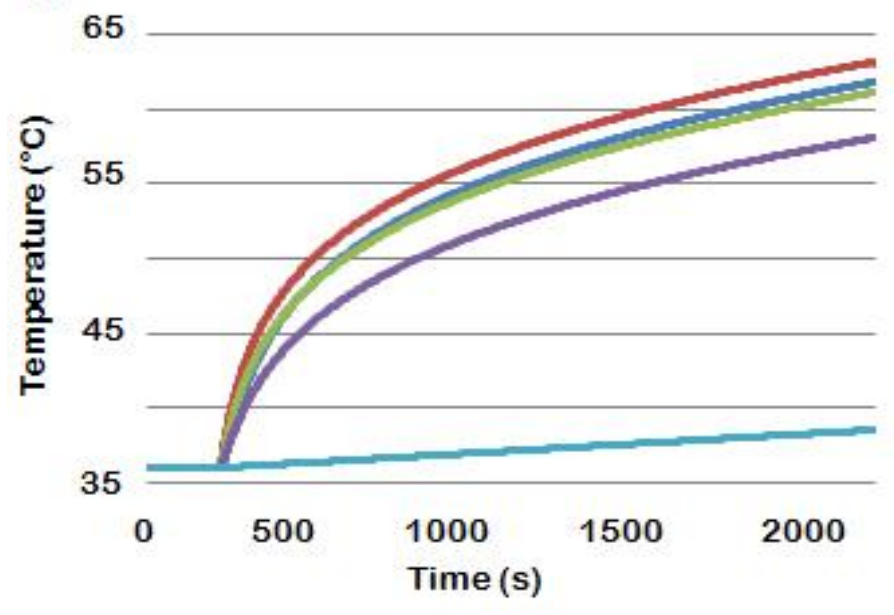

the local increase in power deposition and significantly underestimate the effect of EMF exposure. SAR values computed for lead models of different radius show that the SAR gradient decreases to negligible values at a distance from the tip comparable to the diameter of the lead itself. For a $2 \mathrm{~mm}$-thick wire (value close to the real dimension of a PM or ICD lead), a $10 \mathrm{~g}$ mass results in a cube whose side is more than 10 bigger than the diameter of the lead. If averaged over 0.1 $\mathrm{g}$ mass (i.e. over a $5 \times 5 \times 5 \mathrm{~mm}^{3}$ region), the resulting maximum local SAR at the lead tip is about $90 \%$ greater than the $10 \mathrm{~g}$ averaged SAR. The results of the numerical model we developed show that even the $1 \mathrm{~g}$ averaging mass is too big if compared to the dimensions of the SAR gradient that rises around the wire tip (about $70 \%$ less than the $0.1 \mathrm{~g}$ averaged SAR).

b)
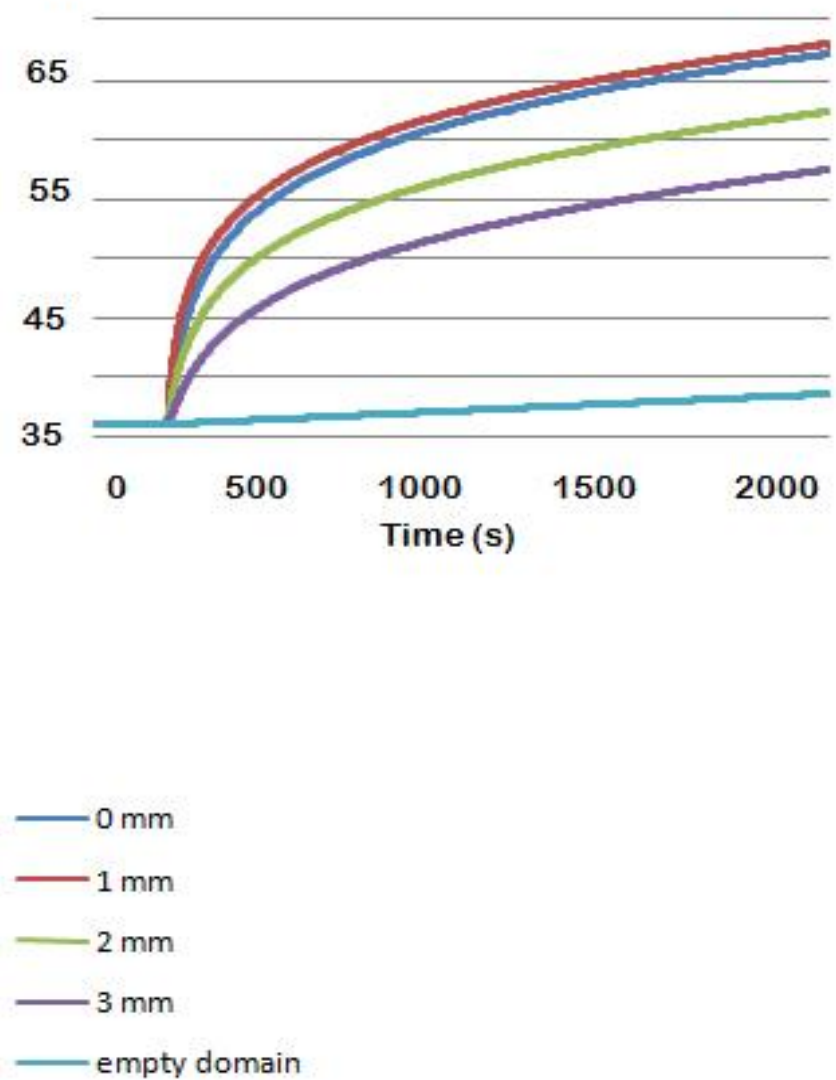
Figure 7. Temperature increase at different distances $(0 \mathrm{~mm}, 1 \mathrm{~mm}, 2 \mathrm{~mm}, 3 \mathrm{~mm})$ from the wire tip: a) $1 \mathrm{~mm}$-thick wire; b) $2 \mathrm{~mm}$-thick wire; c) $4 \mathrm{~mm}$-thick wire. In each graph the temperature increase caused by the RF source inside the empty phantom is also reported.

a)

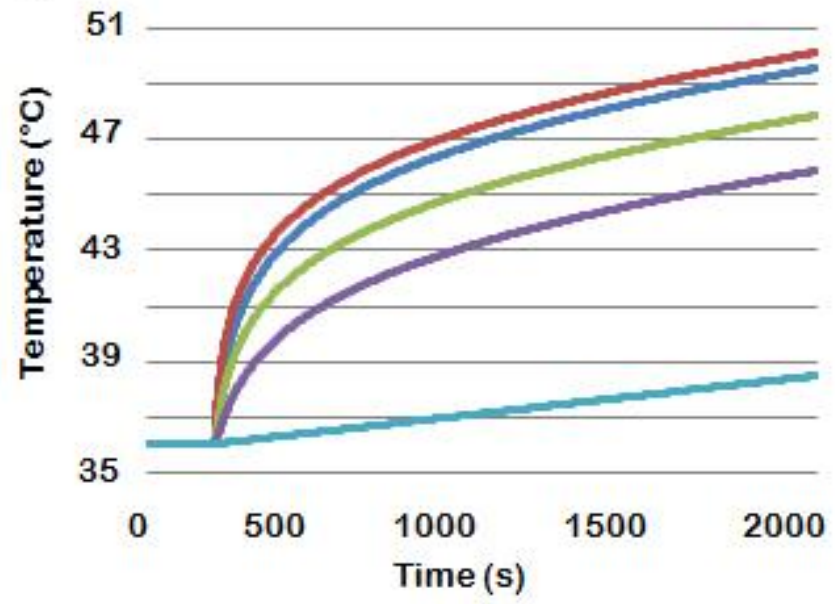

c)

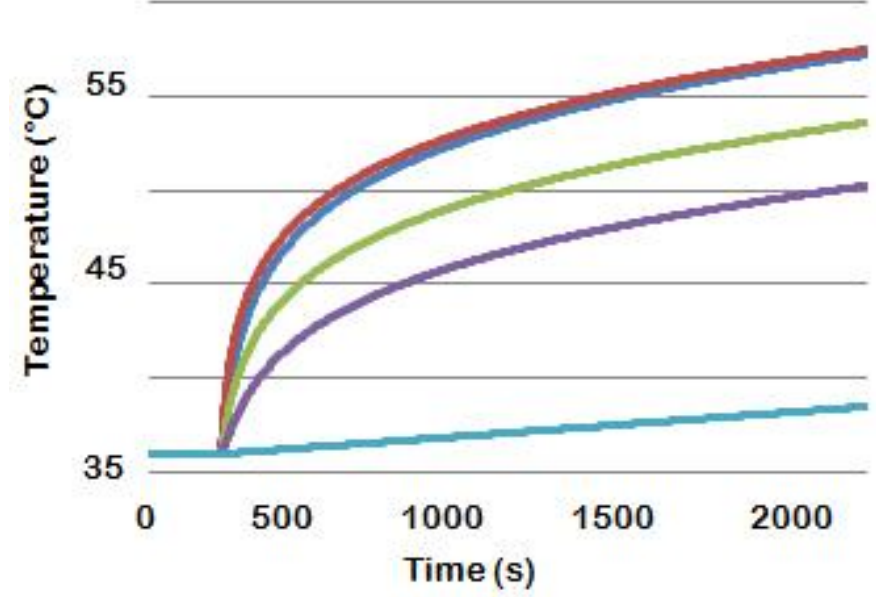

b)

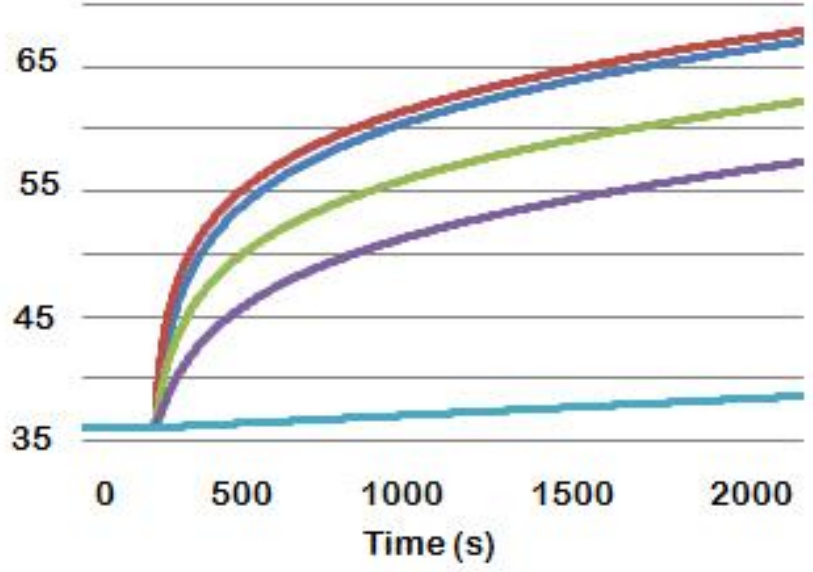

$0 \mathrm{~mm}$

$-1 \mathrm{~mm}$

$-2 \mathrm{~mm}$

$-3 \mathrm{~mm}$

-empty domain

Figure 8. Temperature increase at different distances $(0 \mathrm{~mm}, 1 \mathrm{~mm}, 2 \mathrm{~mm}, 3 \mathrm{~mm})$ from the wire tip: a) $15 \mathrm{~cm}$-long wire; b) $25 \mathrm{~cm}$-long wire; c) $40 \mathrm{~cm}$-long wire. In each graph the temperature increase caused by the RF source inside the empty phantom is also reported.

The comparison between the SAR distributions computed for wires of different thickness and length shows that the diameter of the metallic implant is the element that plays the major role in determining the gradient dimensions: the thicker is the wire, the slower is the exponential decay of the deposited power around the tip. Another aspect that needs to be taken into account is the SAR peak value at the wire tip; however, the SAR gradient that rises around wires of different length have comparable dimensions, in spite of SAR peak values pretty different. It suggests that, more than the length, the thickness of the metallic implant is the discriminate element that must be considered in order to identify the optimal averaging mass for a reliable SAR evaluation.

The localised SAR values we found were significantly higher than the limits defined by the ICNIRP guidelines. Such limits have been chosen for averaging volumes of $1 \mathrm{~g}$ and $10 \mathrm{~g}$, in order not to exceed $1^{\circ} \mathrm{C}$ in temperature rise, threshold beyond which adverse health effects may occur [1]. In our numerical study, SAR was computed 
over smaller volumes and thus our results cannot be immediately related to an increase in local temperature dangerous for the patient. High SAR values involve only very small regions, comparable with the dimension of the implant, and thus are unlikely to cause adverse systemic effects that could compromise the patient safety. However, some considerations still need to be done. In particular, the RF power deposited around the metallic structure might modify the interface between the implant and the biological tissue, causing, for example, a variation in the contact impedance seen by the implant. As a consequence, all the medical devices that require an energy transfer from the implant to the tissue or that need to read biological signals, might not work properly anymore.

The thermal analysis shows that the induced current which flows from the metallic lead inside the dielectric domain produces high temperature increases around the tip. These results are obtained assuming no contribution from convection; the expected induced temperature increase inside real biological tissues perfused with blood would thus be much lower, but some considerations could be still valid: the highest heating is obtained for the thinnest wire, where the electromagnetic flux that arises near the implant is denser than for larger surfaces. As a consequence, the resistive losses inside the dielectric domain are higher, as well as the induced heating. Even if the temperature increases resulting from the numerical study cannot be immediately related to an actual damage for biological tissues, owing to the very simplicity of the model, the values we found suggest in any case that the heating induced by the RF field on implanted patients needs particular attention and further investigation.

In the numerical model, we used as RF source a combination of plane waves at the typical frequencies clinical MRI scanner (i.e. $64 \mathrm{MHz}$ ). However, even if the local SAR values and the temperature increases may vary as a function of the frequency, of implant geometry and position, most of the considerations relating to the SAR evaluation can be reasonably extended to all the $\mathrm{RF}$ range.

\section{Conclusions}

In order to evaluate the thermal effects of RF exposure on patients implanted with electrical active devices, it is necessary to refine the actual measure methods. In particular, present standards choose an averaging mass of $10 \mathrm{~g}$ of tissue to estimate the localised SAR induced by the RF: it would lead to a significant underestimation of the local SAR of the tissues near the implant. The amount of averaging mass should be chosen as a function of the dimension of the implanted object and of the SAR gradient that is generated around it. At the same time, the averaging volume has to be not too small to be just a single "hot spot", caused by the intrinsic errors of the numerical method and not relevant for the evaluation of potential tissue damage due to RF exposure. A good trade-off could be to choose the averaging mass with dimensions doubled than the thickness of the exposed metallic part of the implant.

\section{Study Limitation}

Aim of this study is to show that present standards, which were defined to limit the RF exposure of general public, cannot be adopted also in the particular case of people bearing metallic implants. The model of the implant as well as of the human phantom are far from a realistic representation. However, our simplified model do not substantially affects the validity of the general results we obtained. A more detailed analysis of realistic implant configurations, structures and geometries, could be the object of future studies.

\section{References}

1. International Commission on Non-Ionizing Radiation Protection (ICNIRP) 1998 Guidelines for Limiting Exposure to Timevarying Electric, Magnetic, and Electromagnetic Fields (up to $300 \mathrm{GHz}$ ), Health Physics, 74: 494-522

2. U.S. Department of Health and Human Services, Food and Drug Administration, Center for Devices and Radiological Health. 
Guidance for industry and FDA staff, criteria for significant risk investigations of magnetic resonance diagnostic devices. 2003. 5p.

3. ICNIRP guidelines on limiting exposure to non-ionizing radiation - Protection of the patient undergoing a magnetic resonance examination - Health Physics 6 1(6), 923-9 28; 1991.

4. Luechinger R, Duru F, Zeijlemaker VA, Scheidegger MB, Boesiger $\mathrm{P}$, Candinas $\mathrm{R}$. 2002 Pacemaker reed switch behavior in 0.5 , 1.5 , and 3.0 Tesla magnetic resonance imaging units: are reed switches always closed in strong magnetic fields? Pacing Clin. Electrophysiol. 25:1419-1423.

5. Martin ET, Coman JA, Shellock FG, Pulling CC, Fair R, Jenkins K 2004 Magnetic resonance imaging and cardiac pacemaker safety at 1.5-Tesla. J Am Col Cardiol. 43(7):1315-24

6. Helfer JL, Gray RW, MacDonald SG, Bibens TW: Can pacemakers, neurostimulators, leads, or guide wires be MRI safe? Technological concerns and possible resolutions. Minim Invasive Ther Allied Technol 2006, 15(2):114-20

7. Mattei E, Calcagnini G, Censi F, Triventi M, Bartolini P. Numerical FDTD models of electromagnetic field generated by the RF coil of a MRI scanner: comparison among different solutions. Proceedings of the Sixth IASTED International Conference, Biomedical Engineering, Innsbruck, Austria 2008, ISBN Hardcopy: 978-0-88986-7215/CD 978-0-88986-722-2

8. Mattei E, Triventi M, Calcagnini G, Censi F, Kainz W, Mendoza G, Bassen HI, Bartolini P. Complexity of MRI induced heating on metallic leads: experimental measurements of 374 configurations Biomed Eng Online. 2008 Mar 3;7:11

9. ANSI/AAMI PC69:2000 Active implantable medical devices - Electromagnetic compatibility - EMC test protocols for implantable cardiac pacemakers and implantable cardioverter defibrillators American National Standard

10. Bassen H, Kainz W, Mendoza G, Kellom T 2006 MRI-induced heating of selected thin wire metallic implants-- laboratory and computational studies-- findings and new questions raised. Minim Invasive Ther Allied Technol. 15(2):76-84

11. C95.3-2003: Recommended Practice for Measurements and Computations with Respect to Human Exposure to RadioFrequency Electromagnetic Fields, 100 $\mathrm{kHz}$ to $300 \mathrm{GHz}, 2003$ Institute of Electrical and Electronics Engineers (EEEI)

12. Balzano Q, Garay O, Steel FR 1978 Heating of biological tissue in the induction field of VHF portable radio transmitters IEEE Trans. on Vehicular Technology VT-27 (2): 51-56

13. Chatterjee I, Yong-Gong G, Gandhi OP 1985 Quantification of electromagnetic fields in humans from body-mounted communication receivers, IEEE Transactions Vehicular Technology VT-34 (2): 55-62

14. Cleveland RF, Athey TW 1989 Specific absorption rate (SAR) in models of the human head exposed to hans-held UHF portable radios, Bioelectromagnetics 10: 173-186

15. Kuster N, Balzano Q 1992 Energy absorption mechanisms by biological bodies in the near field of dipole antennas, IEEE Trans. on Vehicular Technology VT-41: 17-23

16. Saunders RD, Kowalczuk CI, Sienkiewicz ZJ 1991 Biological effects of exposure to nonionising electromagnetic fields and radiation, III. Radiofrequency and microwave radiation, Chilton, NRPB-R240 (London, HMSO)

17. Scott J 1988 A finite element model of heat transport in the human eye Phys. Med. Biol. 33: $227-41$ 